\title{
Influence of post relining with composite and association of universal dentin adhesive and dual cure resin cement on fiber post push-out bond strength.
}

\author{
Luís Roberto M. Martins (PQ), Rebeca M. Domingues (IC), Cristiane R. F. Yanikian (PG), e Daniel \\ Sundfeld-Neto (PG).
}

\section{Abstract}

Bond procedures into root canal are still a challenge, therefore, this study evaluated different adhesive approaches in the fiber posts retention. Alternative techniques may be used to retain fiber posts. However, adhesion quality worsening in deeper regions of the root canal.

Key words: Dental cements, cementation, dentin.

\section{Introduction}

The fiber posts luting is a critical procedure, mainly because of difficult access to the root canal and poor light access during the resin cement polymerization. ${ }^{1}$ Therefore, the aim of this study was to evaluate the influence of post relining with composite resin and the association of universal dentin adhesive on fiber posts push-out bond strenght.

\section{Results and Discussion}

The studied adhesive techniques were: G1: post + dentin total-etching (DTE) + Scothbond MultiPurpose (SBMP) + RelyX ARC (ARC); G2: post + Single Bond Universal (SBU) + ARC; G3: post + DTE + SBU + ARC; G4: relined post + DTE + SBMP + ARC; G5: relined post + SBU + ARC; G6: relined post + DTE + SBU + ARC. Ten samples of each group were submitted to push-out test until failure $(50 \mathrm{Kgf}$ at $5 \mathrm{~mm} / \mathrm{min}$ ) and the failure pattern was analyzed by stereomicroscopy (40x). The results are described in Chart 1 and Figure 1 . The split-plot analysis of variance showed that the post relining $(p=0.0954)$ and different adhesive approaches $(p=0.1724)$ did not affect the post retention peformance. However, the greater the depth into root canal, the worse is the fiber post retention. Note that in the control group the predominant failure is adhesive between cement and post and in the experimental groups is adhesive between dentin and resin cement. The decreased dentin bond strength in experimental groups maybe explained by incompatibility between the dual cure resin cement with the universal dentin adhesive acidicity. ${ }^{2}$
Chart 1. Mean and standard deviation of the push-out values (MPa) as a function to the luting technique and the root area.

\begin{tabular}{c|ccc}
\hline \multirow{2}{*}{$\begin{array}{c}\text { Luting } \\
\text { Technique }\end{array}$} & \multicolumn{3}{|c}{ Root canal area } \\
\cline { 2 - 4 } & Cervical & Medium & Apical \\
\hline G1 & $10,49(2,69)^{\mathrm{Aa}}$ & $8,14(1,24)^{\mathrm{ABa}}$ & $5,46(1,81)^{\mathrm{Bb}}$ \\
$\mathbf{G 2}$ & $8,04(1,51)^{\mathrm{Aa}}$ & $6,91(2,00)^{\mathrm{ABb}}$ & $4,01(1,36)^{\mathrm{Bb}}$ \\
$\mathbf{G 3}$ & $9,29(1,98)^{\mathrm{Aa}}$ & $6,28(1,76)^{\mathrm{ABb}}$ & $4,80(2,15)^{\mathrm{Bb}}$ \\
G4 & $11,26(2,35)^{\mathrm{Aa}}$ & $8,91(1,31)^{\mathrm{Aba}}$ & $6,28(2,54)^{\mathrm{Bb}}$ \\
G5 & $9,85(2,22)^{\mathrm{Aa}}$ & $9,50(1,37)^{\mathrm{Aa}}$ & $6,89(2,00)^{\mathrm{Ab}}$ \\
G6 & $11,13(1,95)^{\mathrm{Aa}}$ & $8,99(2,23)^{\mathrm{ABa}}$ & $6,01(1,26)^{\mathrm{Bb}}$ \\
\hline
\end{tabular}

* Uppercase letters indicate statistical differences between rows and lowercase letters indicate statistical differences between columns $(p<0.05)$, according to Tukey's HSD test.

Figure 1. Failure pattern distribution.

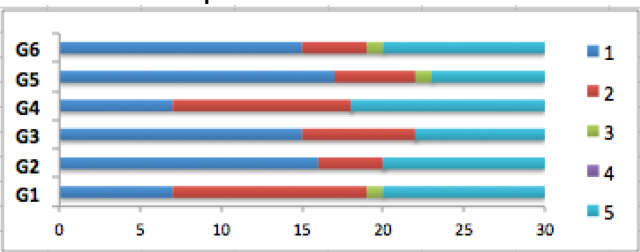

* 1) Adhesive failure between dentin and resin cement; 2) Adhesive failure between resin cement and fiber post; 3) Resin cement cohesive failure; 4) Fiber post cohesive failure and; 5) mixed failure.

\section{Conclusions}

The studied alternatives techniques can be used for cementation of fiber posts. However, the bond quality in deeper root canal regions is still a challenge to be overcome.

\section{Acknowledgement}

This study was suported by PIBIC/CNPq.

\footnotetext{
Faria-e-Silva, A. L.; Arias, V. G.; Soares, L. E.; Martin A. A. e Martins, L. R. J. Endod. 2007, 33, 303-305.

${ }^{2}$ Cheong, C.; King, N. M.; Pashley, D. H., Ferrari, M.; Toledano, M. e Tay, F. R. Oper. Dent. 2003, 28, 747-755.
} 